\title{
Effects of transplanted GDNF gene modified marrow stromal cells on focal cerebral ischemia in rats
}

\author{
Yunliang Wang ${ }^{1}$, Tongchao Geng ${ }^{2}$, Amanda $\mathrm{Ni}^{3}$, Honglei Yin ${ }^{1}$ and Bing Han ${ }^{1}$ \\ 1 Department of Neurology, No. 148 Hospital of PLA, Zibo, Shandong, China \\ ${ }^{2}$ Department of Neurology, Yuquan Hospital Affiliated to Tsinghua University, Beijing, China \\ ${ }^{3}$ Northwestern University, Evanston, IL, USA
}

\section{Edited by:}

Hongyun Huang, Neuroscience

Institute of Taishan Medical

University, China

Reviewed by:

Ying Li, Institute of Neurology, UK Shinn-Zong Lin, China Medical

University Hospital, Taiwan

\section{*Correspondence:}

Yunliang Wang, Department of

Neurology, No. 148 Hospital of PLA,

Zibo, Shandong 255300, China.

e-mail:wangyunliang81@163.com

\begin{abstract}
Objective: To evaluate the therapeutic effect of transplanted glial cell derived neurotrophic factor (GDNF) modified marrow stromal cells (MSCs) on an experimental ischemic brain injury based on the behavioral, morphological, and immunohistochemical observations. Methods: The MSCs from four-week newborn rats were cultured in vitro. The cerebral ischemia and reperfusion model was established in adult Sprague-Dawley (SD) rats by using the suture method. Three days after model establishment, the animals were injected with prepared MSCs via their caudal veins. The animals were then divided into a sham-operation group, ischemia group, MSCs transplantation group, or GDNF + MSCs transplantation group and were scored for their neurobehavioral manifestations at 3, 14, and 28 days after the transplantation was performed. At this time, the survival condition of intracerebral transplanted cells was measured by laser confocal microscopy while the effect of transplantation on the GDNF expression in the ischemic brain tissue was evaluated. Results: The MSCs cells transfected with GDNF gene were characterized by green fluorescence. Three days after the transplantation, the animals that underwent the cell transplantation showed significantly better behavioral data than the controls. Fourteen days after transplantation, the animals transplanted with GDNF gene modified MSCs were better than those transplanted with common MSCs. As compared with common MSCs transplantation, GDNF + MSCs transplantation was significantly more effective in reducing apoptotic cell volume and enhancing Bcl-2 expression, which was favorable for the ischemic brain injury. Conclusions: Transplanted GDNF modified MSCs can improve the nervous function and have a protective effect on the ischemic brain injury through reducing apoptotic cell volume and enhancing the expression of anti-apoptotic gene $\mathrm{Bcl}-2$.
\end{abstract}

Keywords: marrow stromal cells (MSCs), glial cell derived neurotrophic factor (GDNF), cerebral ischemia, Bcl-2, apoptosis

\section{INTRODUCTION}

As a principle component of non-hematopoietic system in bone marrow, marrow stromal cells (MSCs) (Shichinohe et al., 2008; Zacharek et al., 2010; Yilmaz et al., 2011) can express multiple neuron- and gliocyte-specific markers and grow morphological processes similar to that of neural cells when treated with inducers in vitro. This indicates the potential of MSCs to differentiate into neural cells and thus provides theoretical evidence for cell transplantation in treating ischemic brain injuries. Glial cell derived neurotrophic factor (GDNF) (Oppenheim et al., 1995) is a potent neurotrophic factor, playing an important role in the differentiation, development, growth, and survival of the cells in the central nervous system.

In the present study, MSCs were cultured using the adherence method. The expression vector was constructed after the GDNF gene was cloned from rats. The MSCs cultured in vitro were transfected with cationic liposome-mediated eukaryotic expression pDNA3-GDNF. The expressed green fluorescent protein was considered the reporter gene to optimize the transfection condition.
The transgenic MSCs obtained through screening were considered as the cell transplantation tracer. The focal brain ischemia model was established in rats by using the suture method and was transplanted with GDNF modified MSCs. The apoptotic cell volume and expression of Bcl-2 before and after the transplantation were observed in order to evaluate the mechanisms of the protective effect of the exogenous stem cell and the neurotrophic factor on brain ischemia. This will provide theoretical evidence for the clinical application of cell and gene therapy for ischemic cerebrovascular diseases.

\section{MATERIALS AND METHODS ANIMALS AND REAGENTS}

Adult Sprague-Dawley (SD) rats, male and female, weighing between $250 \mathrm{~g}$ and $300 \mathrm{~g}$, and four-week-old newborn rats were purchased from Laboratory Animal Center of Chongqing Medical University. Rabbit anti-Bcl-2 antibody was purchased from Jingmei (Shanghai, China). Cationic liposome-mediated eukaryotic expression pDNA3-GDNF plasmid (constructed in 
our laboratory) carrying green fluorescent protein was used to transfect MSCs cultured in vitro.

\section{ANIMAL MODEL}

Right middle cerebral artery occlusion (MCAO) was induced with an intraluminal filament as previously reported (Longa et al., 1989). Transient right MCAO was induced for two hours by advancing a $40 \mathrm{~mm}$ length monofilament nylon suture with its tip rounded by heating near a flame to block the origin of the MCA. Reperfusion was performed by withdrawal of the suture two hours after MCAO. Animals subjected to sham surgery were treated similarly, except that the filament was not advanced to the origin of the MCA. Rats that failed to exhibit neurological abnormalities at two hours after stroke were excluded from this study.

\section{CULTURING AND IDENTIFICATION OF MSCs FROM NEWBORN RATS AND GDNF GENE TRANSFECTION}

The MSCs from newborn rats were successfully cultured using the adherence method after serial passage. Four-week-old SD rats received the left hindquarter amputation. The rat bone marrow was harvested from femur and tibia that were just amputated. Flushed out the bone marrow, separated the mononuclear cell layer, add the culture liquid $\alpha$-Minimal Essential Medium (MEM) which contains $10 \%$ serum of fetal cow to the cell-floating liquid and plant them in the culture instruments in the condition of $37^{\circ} \mathrm{C}$ and $5 \% \mathrm{CO}_{2}$ for the primary generation culture. The third and fourth generation cells grow well, and the amount cells more than primary generation.

The GDNF gene expression vector was constructed after the gene was cloned from rats. The MSCs cultured in vitro were transfected with pDNA3-GDNF which was expressed by eukaryocytes under the mediation of cationic liposome. The expressed green fluorescent protein was considered the reporter gene in order to optimize the transfection condition. The transgenic MSCs obtained through screening were considered the cell transplantation tracers.

\section{ANIMAL GROUPING}

The animals with related nervous symptoms were divided randomly into three groups: untreated ischemia group (Group A), MSCs transplantation group (Group B), and MSCs + GDNF group (Group C). Each group included 30 animals used for the evaluation at three separate time points $(3,14$, and 28 days after transplantation).

\section{PREPARATION OF DONOR CELL SUSPENSION AND TRANSPLANTATION PROCEDURE}

The well-grown MSCs previously transplanted with pcDNA3GDNF-GFP plasmid were digested into monoplast suspension with $0.25 \%$ trypsin and centrifuged at $800 \mathrm{rpm}$ for five minutes. The precipitated cells were re-suspended in $500 \mu \mathrm{l}$ normal saline and gently beat with a pipette before they were transferred into an Eppendorf (EP) tube where they were centrifuged at $800 \mathrm{rpm}$ for another five minutes. The precipitated cells were then resuspended in $100 \mu \mathrm{l}$ normal saline and incubated at $37^{\circ} \mathrm{C}$ in a $5 \% \mathrm{CO}_{2}$ incubator to be stored for use. Before use, the cells were centrifuged again and the final concentration of the cells obtained was $5 \times 10^{6} / \mathrm{ml}$. The transplantation was conducted intravenously via the bilateral caudal veins at 72 hours after ischemia model was successfully established. Each animal in GDNF + MSCs transplantation group (Group C) was injected with $500 \mu \mathrm{l}$ of cell suspension. The syringe needle was slowly withdrawn before after being retained in the vein for two minutes. The animals in MSCs transplantation group (Group B) were injected with $500 \mu \mathrm{l}$ of common MSCs suspension. The animals in untreated ischemia group (Group A) were injected with same volume of sterilized normal saline via the same route.

\section{FUNCTIONAL TESTS}

A behavioral rating scale to grade the extent of injury was modified from published scales (Bederson et al., 1986). Three behaviors were tested: ipsilateral circling, bilateral grasp, and beam walking. The extent of circling to the side of the infusion was graded from 0 (no circling) to 4 (always circled). Grasp was tested by placing the rat's paws on the edge of a box 14 inches high; strength of the hemiparetic paw was graded from 0 (grasped well) to 4 (unable to grasp with forepaw). Beam walking was graded by placing each rat on a beam; a grade of 0 indicated that it easily traversed the beam, while a grade of 4 was given those unable to walk on the beam. A total injury score was calculated as the sum of the grades on the three tests. The higher score, the more severe the injury.

\section{SPECIMEN COLLECTION AND IMMUNOHISTOCHEMICAL EXAMINATION}

At 3,14, and 28 days after transplantation, the animals were terminated. Their brain tissue was fixed using normal saline and $4 \%$ paraformaldehyde through cardiac perfusion. Each half portion of the specimen was used to prepare frozen and paraffin embedded coronal sections $(3 \mu \mathrm{m})$. Every five successive sections were selected from every 200 sections. The sections selected were deparaffinaged, treated in microwave for antigen repair, added with rabbit anti-Bcl-2 antibody (1:100, Santa Cruz) and incubated at $4^{\circ} \mathrm{C}$ overnight. After the secondary antibody was added, they were treated with chromogenic agent DAB, dehydrated, cleared, and sealed. The replacement of primary antibody by PBS was used in the negative control. Under microscope, the positive cell was characterized by brown, stained cytoplasm.

\section{TUNEL ASSAY}

After the sections were deparaffinaged as per usual, protease $\mathrm{K}$ was added and the sections were incubated in a $25^{\circ} \mathrm{C}$ wet box for 30 minutes. Then, each of the sections was added with $50 \mu \mathrm{l}$ of TUNEL mixed solution (The TUNEL kit was purchased from Tongfang [Beijing, China]) and incubated in a $25^{\circ} \mathrm{C}$ wet box for 60 minutes. After that, they were added with POD, $50 \mu \mathrm{l} /$ section, for transformation, and incubated in a $25^{\circ} \mathrm{C}$ wet box for 30 minutes. The sections were then treated with chromogenic DAB, dehydrated, cleared, and sealed. In the negative control, the TUNEL mixed solution was replaced by a labeling solution that did not contain large Klenow fragments. The positive cell was characterized by a brown stained nucleus with clear background. Under microscope, the positive cells in the graticule field were counted by using the micrometer of the $16 \mathrm{D}$ eyepiece 
while the values obtained were averaged as the positive cell density of each animal.

\section{STATISTICAL MANAGEMENT}

The database and corresponding statistical analyses were performed using statistical software SPSS 10.0. T-test, ANOVA, and LSD analysis were used in the comparison between groups. $p<0.05$ was considered to be statistically significant.

\section{RESULTS \\ IN VITRO CULTURING, IDENTIFICATION AND GDNF GENE MODIFICATION OF RAT MSCs}

The MSCs became adherent and grew into colonies with extended and enlarged processes at 4-5 days after primary culture (Figure 1). The modified MSCs were obtained when the common MSCs were transfected with well-constructed pcDNA3-GDNF plasmid mediated by cationic liposome. Microscopically, the nuclei and cytoplasm of the differentiated cells showed green fluorescence indicating a successful transfection. Fourteen days after transplantation, the number of fluorescent cells in MCSs-pcDNAGDNF-GFP group was greater than that of the cells in MSCspcDNA-GFP group. The fluorescent cells in the former group also had better configuration and stronger fluorescence intensity. Twenty-eight days after transplantation, the morphologically regular fluorescent cells in the MCSs-pcDNA-GDNF-GFP group were distributed extensively in the brain tissue (Figure 2).

\section{NEUROBEHAVIORAL SCORING FINDINGS}

The animals in all groups showed obvious cerebral ischemic symptoms after the experimental model was established. At three days after transplantation, both the animals in the MSCs

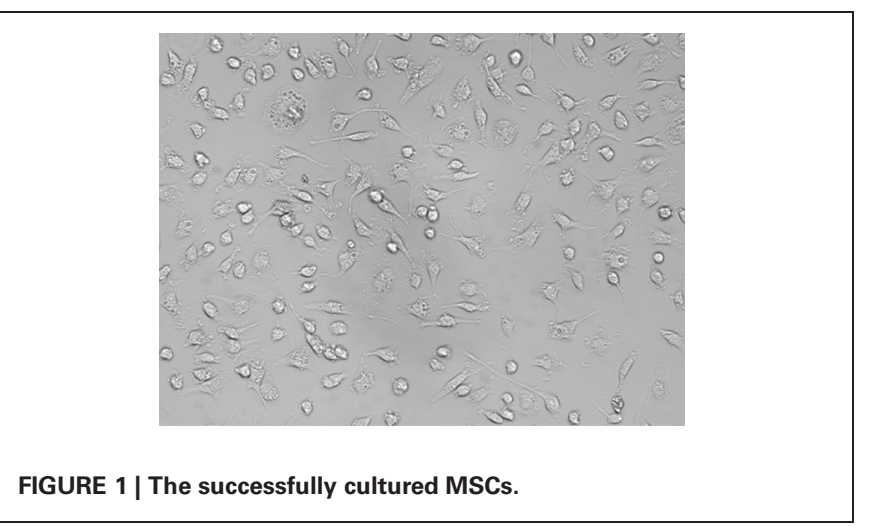

transplantation group and MSCs + GDNF transplantation group had significantly better neurobehavioral scores than those in the ischemia group, suggesting the effect of transplanted MSCs on the nervous function of the rats with cerebral infarction. Meanwhile, three days after the transplantation, the animals in the MSCs + GDNF transplantation group had a mildly better neurobehavioral scores than those in MSCs transplantation group $(7.27 \pm 1.25$ vs. $6.84 \pm 1.12)$. And, 14 and 28 days after transplantation, the behavioral score of the MSCs + GDNF transplantation group was significantly better than that of the MSCs transplantation group, suggesting the superiority of GDNF modified MSCs transplantation (See Table 1).

\section{TUNEL FINDINGS}

Three days after transplantation, the MSCs group and MSCs + GDNF group had fewer apoptotic cells than the ischemia group $(P<0.05)$, but similar apoptotic cell volume. The value of apoptotic cells in Groups A, B, and C were $76 \pm 7.44$, $68 \pm 8.19$, and $65 \pm 8.81$, respectively. After transplantation, the value decreased to $51 \pm 8.27,42 \pm 6.28$, and $35 \pm 4.06$ at day 14 and $33 \pm 9.31,24 \pm 7.35$, and $18 \pm 6.85$ at day 28. From the data, it could be seen that the TUNEL positive cells decreased more significantly in the MSCs group and the MSCs+ GDNF group $(P<0.05)$. Moreover, the apoptotic cells located at the cortex around the ischemic region in the MSCs + GDNF group were significantly fewer than those in the MSCs group, suggesting that the transplanted GDNF modified MSCs could conspicuously suppress the apoptosis of neurons (Figures 3, 4, and 5).

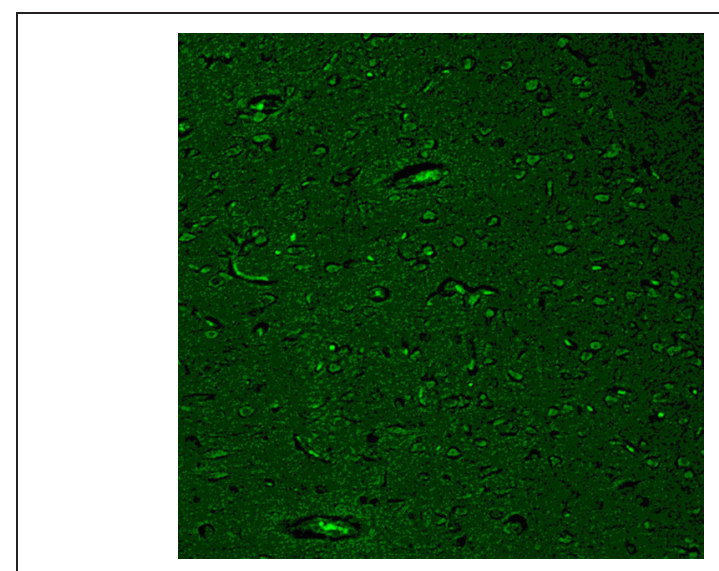

FIGURE 2 | Findings at 28 days after GDNF + MSCs transplantation.

Table 1 | The neurobehavioral scores in each group rats at different time points $(\bar{x} \pm \mathbf{s})$.

\begin{tabular}{llllr}
\hline Group & $\begin{array}{l}\text { Before } \\
\text { transplantation }\end{array}$ & $\begin{array}{l}\text { Three days after } \\
\text { transplantation }\end{array}$ & $\begin{array}{l}\text { 14 days after } \\
\text { transplantation }\end{array}$ & $\begin{array}{l}\mathbf{2 8} \text { days after } \\
\text { transplantation }\end{array}$ \\
\hline Untreated ischemia group (Group A) & $9.02 \pm 1.62$ & $9.06 \pm 1.44^{a}$ & $5.78 \pm 1.33^{a}$ & $4.65 \pm 0.82^{a}$ \\
MSCs transplantation group (Group B) & $8.67 \pm 0.98$ & $7.27 \pm 1.25^{b}$ & $4.59 \pm 0.76^{b}$ & $3.32 \pm 0.41^{b}$ \\
MSCs + GDNF group (Group C) & $8.84 \pm 1.13$ & $6.84 \pm 1.12^{c}$ & $3.42 \pm 0.66^{c *}$ & $2.02 \pm 0.27^{c *}$ \\
\hline
\end{tabular}

${ }^{b, c}$ vs. ${ }^{a} P<0.05$

${ }^{*} v s .{ }^{b} P<0.05$. 


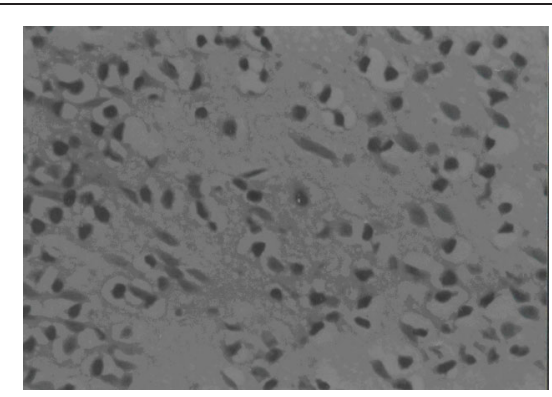

FIGURE 3 | TUNEL findings of the ischemia group at 28 days after sham transplantation.

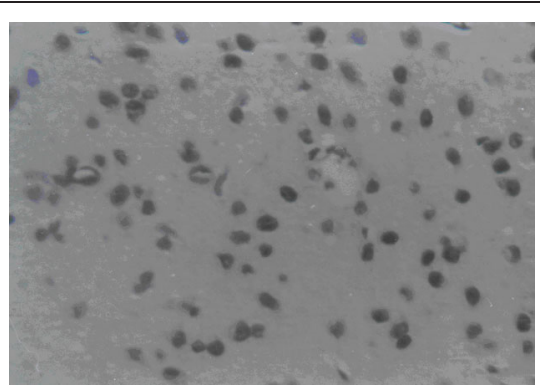

FIGURE 4 | TUNEL findings of MSCs group at 28 days after transplantation.

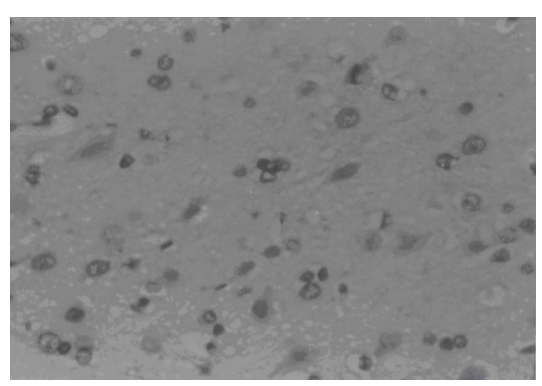

FIGURE 5 | TUNEL findings of GDNF + MSCs group at 28 days after transplantation.

\section{IMMUNOHISTOCHEMICAL FINDINGS FOR BcI-2}

Three days after transplantation, the number of Bcl-2 positive cells in the MSCs group and the MSCs + GDNF group was greater than that in the ischemia group $(P<0.01)$. The positive cells were characterized by a brown color and irregular appearances such as round-shaped cells, spindle-shaped cells, or the presence of long processes. It was observed that the number of Bcl-2 positive cells between the MSCs group and the MSCs + GDNF group was not different from each other. However, 14 days after the transplantation, the MSCs + GDNF group had significantly more Bcl-2 positive cells than the MSCs group $(20.54 \pm 2.27,39.28 \pm 3.28$, and $45.33 \pm 4.06, P<0.05)$ (Figures 6 and 7).

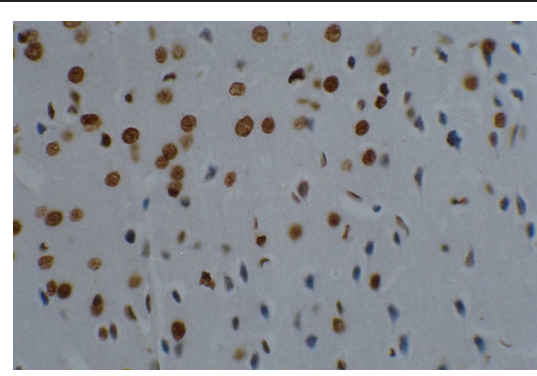

FIGURE 6 | Immunohistochemical findings of MSCs group at 14 days after transplantation.

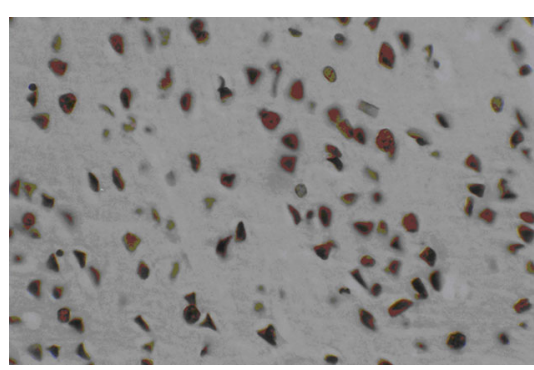

FIGURE 7 | Immunohistochemical findings of GDNF + MSCs group at 14 days after transplantation.

\section{DISCUSSION}

Under the current situation of the application of gene therapy for central nervous diseases, it is necessary to find a method by which the stromal cells can be amplified in large volume through in vivo within a short time period. As a confirmed cell source with differentiation potential to nervous tissue, MSCs are expected to be used for the treatment of diverse nervous diseases because they are not only present with conspicuous advantages such as autologous origin and easy harvesting, but they are also absent from the ethical and immunological rejection problems that fetal stem cell transplantation often develops (Dawn and Bolli, 2005; Wang et al., 2005). Recent studies showed that (GDNF) had a nutritive effect not only on dopaminergic neurons but also on many other central and peripheral motor, sensory, and sympathetic neurons, functioning to promote the growth, differentiation, and repair of neurons. It also plays an important role in protecting neurons from injury and in the repair, regeneration and axon formation of an injured neuron (Oppenheim et al., 1995; Sauer et al., 1996; Wang et al., 2011). As its irreplaceable role in the nourishment of nervous system, GDNF currently is a hot spot in the research of neurology.

It was reported that if the ischemic rats were intracerebroventricularly or intraparenchymally administered with exogenous GDNF protein or adenovirus-mediated GDNF gene, their motor, sensory, and recognition function would improve greatly, their cerebral edema scope, apoptotic cell number, and infarction volume would decrease and the regenerated striatal neurons would increase (Kobayashi et al., 2006; Androutsellis-Theotokis et al., 2008). Further studies showed that GDNF could antagonize 
injury induced by reactive oxygen and free radicals through upregulating the expression of glutathione peroxidase, hydrogen peroxidase, and superoxide dismutase (SOD). GDNF could also reduce the calcium reflux induced by NMDA, a glutamic receptor, via the mitogen activated protein kinase (MAPK) pathway. Therefore, it was reported that GDNF had a protective effect on the ischemic brain injury by suppressing the production of free radicals and active toxics (Nicole et al., 2001; Horita et al., 2006). The above-mentioned studies showed that GDNF could inhibit the development of ischemic brain injury via multiple mechanisms, serving as a potent neuroprotective agent for cerebral ischemia. The key of this present study was the construction of stem cells that were able to secrete GDNF from MSCs previously transfected with pcDNA3-GDNF-GFP expression vector. The GDNF modified MSCs transplantation was not only free from the problems caused by simple GDNF administration such as low local concentration, short half-life, infection potential caused by repeated administration and insufficient expression of plasmids that carry GDNF gene, but also absent of the defects present in the common stem cell transplantation including the apoptosis or early death of the transplanted cells due to a shortage of nutrition.

In the present study, it was revealed that three days after the animal model was established, a vast number of TUNEL positive apoptotic cells appeared at the cortex surrounding the infarcted region. Transplanted MSCs could reduce the production of apoptotic cells which would be beneficial to the cerebral tissue repair and nervous function improvement posterior to ischemia. Its mechanism is probably related to the partial replacement of the apoptotic cells by the transplanted MSCs and to the secretion of GDNF which could alleviate cellular apoptosis. The study further showed that at 14 and 28 days after transplantation, the apoptotic cells present around the ischemic region in the MSCs + GDNF transplantation group were significantly fewer than those in the MSCs group. The Bcl-2 gene is an apoptosis suppressor. Its expression product is mainly located in the mitochondrial, reticular, and nuclear membranes. The major mechanism of the Bcl-2 protein in apoptosis inhibition is the suppression of the production of free radicals and intracellular calcium overloading, reduction of the permeability of mitochondrial membrane, blocking of the release of cytochrome $\mathrm{C}$, and inhibition the activation of

\section{REFERENCES}

Albrecht, S., Bourdeau, M., Bennett, D., Mufson, E. J., Bhattacharjee, M., and LeBlanc, A. C. (2007). Activation of caspase- 6 in aging and mild cognitive impairment. Am. J. Pathol. 170, 1200-1209.

Androutsellis-Theotokis, A., Rueger, M. A., Mkhikian, H., Korb, E., and McKay, R. D. (2008). Signaling pathways controlling neural stem cells slow progressive brain disease. Cold Spring Harb. Symp. Quant. Biol. 73, 403-410.

Bederson, J. B., Pitts, L. H., Tsuji, M., Nishimura, M. C., Davis, R. L., and Bartkowski, H. (1986). Rat middle cerebral artery occlusion: evaluation of the model and development of a neurologic examination. Stroke 17, 472-476.

Dawn, B., and Bolli, R. (2005). Adult bone marrow-derived cells: regenerative potential, plasticity, and tissue commitment. Basic Res. Cardiol. 100, 494-503.

Gage, F. H., Coates, P. W., Palmer, T. D., Kuhn, H. G., Fisher, L. J., Suhonen, J. O., Peterson, D. A., Suhr, S. T., and Ray, J. (1995). Survival and differentiation of adult neuronal progenitor cells transplanted to the adult brain. Proc. Natl. Acad. Sci. U.S.A. 92, 11879-11883.

caspase (Zhan et al., 2001; Albrecht et al., 2007). MSCs transplantation could up-regulate Bcl-2 expression. Fourteen days after transplantation, the MSCs + GDNF group had more Bcl-2 positive cells than the MSCs group suggesting that the MSCs + GDNF transplantation was preferable over the MSCs transplantation in reducing apoptotic cell volume and enhancing Bcl-2 expression, thus playing a more important role in neural function recovery after an ischemic brain injury. The possible reason for this may be that the combined application of MSCs and GDNF up-regulated the expression of anti-apoptotic proteins and consequently inhibited the cascade reaction during ischemia (Lasala et al., 2010).

From the above-mentioned discussion, it can be concluded that the GDNF modified MSCs transplantation is more useful than common the MSCs transplantation in nervous function recovery after an ischemic brain injury. Generally, the MSCs themselves can secrete nerve growth and nutrition factors, but the secreted volume is too small to meet the needs of neural cell repair (Mahmood et al., 2004). After GDNF transfection, the continuously secreted neurotrophic factors can not only improve the survival of transplanted MSCs, but also nourish and partially restore the remaining nerve tissue at the ischemic region. This may contribute to the more favorable efficacy of MSCs + GDNF transplantation. In previous study (Gage et al., 1995), local intracerebral injection induces local brain damage, and particularly, multiple injections may not be clinically acceptable. In the study, the GDNF modified MSCs delivered to ischemic tissue through an intravenous route can not only function to replace the inherent injured cerebral cells similar to MSCs, but also can improve the survival and migration of the transplanted MCSs, promote the formation of collateral circulation, improve the local microenvironment and accelerate the recovery of nervous function through secreted GDNF. This simple approach for cell therapy, which does not necessitate invasive stereotaxic operations, could potentially target pathological sites in a number of brain disorders. The following study will focus on the functionality of transplanted cells, the effect of transplantation on the immune system, and the detailed mechanism of the activation of signal pathway during the recovery of nervous function, in order to provide evidence for the future application of stem cell transplantation in the cerebral ischemic stroke.

Horita, Y., Honmou, O., Harada, K., Houkin, K., Hamada, H., and Kocsis, J. D. (2006). Intravenous administration of glial cell linederived neurotrophic factor genemodified human mesenchymal stem cells protects against injury in a cerebral ischemia model in the adult rat. J. Neurosci. Res. 84 , 1495-1504.

Kobayashi, T., Ahlenius, H., Thored, P., Kobayashi, R., Kokaia, Z., and Lindvall, O. (2006). Intracerebral infusion of glial cell line-derived neurotrophic factor promotes striatal neurogenesis after stroke in adult rats. Stroke 37, 2361-2367.
Lasala, G. P., Silva, J. A., Gardner, P. A., and Minguell, J. J. (2010) Combination stem cell therapy for the treatment of severe limb ischemia: safety and efficacy analysis. Angiology 61, 551-556.

Longa, E. Z., Weinstein, P. R., Carlson, S., and Cummins, R. (1989). Reversible middle cerebral artery occlusion without craniectomy in rats. Stroke 20, 84-91.

Mahmood, A., Lu, D., and Chopp, M. (2004). Intravenous administration of marrow stromal cells (MSCs) increases the expression of growth factors in rat brain after traumatic 
brain injury. J. Neurotrauma 21, 33-39.

Nicole, O., Ali, C., Docagne, F., Plawinski, L., MacKenzie, E. T., Vivien, D., and Buisson, A. (2001). Neuroprotection mediated by glial cell line-derived neurotrophic factor: involvement of a reduction of NMDA-induced calcium influx by the mitogen-activated protein kinase pathway. J. Neurosci. 21, 3024-3038.

Oppenheim, R. W., Houenou, L. J., Johnson, J. E., Lin, L. F., Li, L., Lo, A. C., Newsome, A. L., Prevette, D. M., and Wang, S. (1995). Developing motor neurons rescued from programmed and axotomy-induced cell death by GDNF. Nature 373, 344-346.

Sauer, H., Rosenblad, C., and Bjoklund, A. (1996). GDNF not TGF- $\beta 3$ prevents delayed degeneration of nigral dopaminergic neurons following striatal 6-hydroxydopamine lesion. Proc. Natl. Acad. Sci. U.S.A. 92, 8935-8939.

Shichinohe, H., Kuroda, S., Tsuji, S., Yamaguchi, S., Yano, S., Lee, J. B., Kobayashi, H., Kikuchi, S., Hida, K., and Iwasaki, Y. (2008). Bone marrow stromal cells promote neurite extension in organotypic spinal cord slice: significance for cell transplantation therapy. Neurorehabil. Neural Repair 22, 447-457.

Wang, X., Hou, Z., Yuan, Y., Hou, G., Liu, Y., Li, H., and Zhang, Z. (2011). Association study between plasma GDNF and cognitive function in late-onset depression. J. Affect. Disord. 132, 418-421.

Wang, Y., Deng, Z., Lai, X., and Tu, W. (2005). Differentiation of human bone marrow stromal cells into neural-like cells induced by sodium ferulate in vitro. Cell. Mol. Immunol. 2, 225-229.
Yilmaz, G., Vital, S., Yilmaz, C. E., Stokes, K. Y., Alexander, J. S., and Granger, D. N. (2011). Selectinmediated recruitment of bone marrow stromal cells in the postischemic cerebral microvasculature. Stroke 42, 806-811.

Zacharek, A., Shehadah, A., Chen, J., Cui, X., Roberts, C., Lu, M., and Chopp, M. (2010). Comparison of bone marrow stromal cells derived from stroke and normal rats for stroke treatment. Stroke 41, 524-530.

Zhan, R. Z., Wu, C., Fujihara, H., Taga, K., Qi, S., Naito, M., and Shimoji, K. (2001). Both caspase-dependent and caspase-independent pathways may be involved in hippocampal CA1 neuronal death because of loss of cytochrome $\mathrm{C}$ from mitochondria in a rat forebrain ischemia model. J. Cereb. Blood Flow Metab. 21, 529-540.
Conflict of Interest Statement: The authors declare that the research was conducted in the absence of any commercial or financial relationships that could be construed as a potential conflict of interest.

Received: 15 September 2011; accepted: 07 December 2011; published online: 22 December 2011.

Citation: Wang Y, Geng T, Ni A, Yin H and Han B (2011) Effects of transplanted GDNF gene modified marrow stromal cells on focal cerebral ischemia in rats. Front. Integr. Neurosci. 5:89. doi: 10.3389/fnint.2011.00089

Copyright (c) 2011 Wang, Geng, Ni, Yin and Han. This is an open-access article distributed under the terms of the Creative Commons Attribution Non Commercial License, which permits noncommercial use, distribution, and reproduction in other forums, provided the original authors and source are credited. 